\title{
O uso da maconha (Cannabis sativa L.) na indústria farmacêutica: uma revisão
}

\author{
The use of marijuana (Cannabis sativa $\mathrm{L}_{\text {. }}$ ) in the pharmaceutical industry: a review \\ El uso de marihuana (Cannabis sativa L.) en la industria farmacéutica: una revisión
}

Recebido: 25/08/2021 | Revisado: 08/09/2021 | Aceito: 10/09/2021 | Publicado: 13/09/2021

\author{
Amanda Alves de Lima \\ ORCID: https://orcid.org/0000-0003-0385-2033 \\ Faculdade Integrada Carajás, Brasil \\ E-mail: amandaalves777733@gmail.com \\ Ueslane Coelho Alexandre \\ ORCID: https://orcid.org/0000-0003-1644-440X \\ Faculdade Integrada Carajás, Brasil \\ E-mail: ueslane15@gmail.com \\ Jânio Sousa Santos \\ ORCID: https://orcid.org/0000-0003-2180-1109 \\ Faculdade Integrada Carajás, Brasil \\ E-mail: santosjs.food@gmail.com
}

\begin{abstract}
Resumo
O objetivo do presente estudo foi expor por meio de uma revisão integrativa da literatura a importância do uso dos princípios ativos advindos da maconha (Cannabis sativa) na indústria farmacêutica. Para tal uma revisão integrativa de literatura foi realizada. Adotou-se como critério que incluam documentos de cunho cientifico, que apresentou relação com a aplicação da maconha na indústria farmacêutica em um recorte temporal entre 2006 a 2021 , como intuito em discorrer sobre as evidências científicas dos últimos quinze anos. A utilização dos compostos ativos da Cannabis sativa na indústria ainda é algo pouco explorado, principalmente no Brasil. Um dos principais fatores de que contribui para que não haja maior interesse em torno da utilização da maconha é o uso recreativo ilícito da mesma. Mas esta está sendo liberada para cultivo e estudos de empresas competentes, podendo trazer uma série de benefícios tanto a saúde humana, quando para as indústrias farmacêuticas. A mesma apresenta numerosas ações terapêuticas relacionadas aos metabolitos secundários. É valido ressaltar os efeitos comprovados frente a doenças como epilepsia, ansiedade, depressão, doença de Parkinson, esclerose múltipla e cefaleia. Por esses motivos em concordância com a tendência mundial a qual é notório que os consumidores estão priorizando o uso de produtos à base natural, mostrando o amplo campo de estudo e tecnológicos para desenvolvimentos de estudos posteriores.
\end{abstract}

Palavras-chave: Maconha medicinal; Cannabis; Princípio ativo; Fitoterápicos; Revisão integrativa.

\begin{abstract}
The aim of the present study was to expose, through an integrative literature review, the importance of using active principles derived from marijuana (Cannabis sativa) in the pharmaceutical industry. An integrative literature review was carried out. It was adopted as a criterion that include scientific documents, which presented a relationship with the application of marijuana in the pharmaceutical industry in a time frame between 2006 and 2021, in order to discuss the scientific evidence of the last fifteen years. The use of active compounds from Cannabis sativa in the industry is still something little explored, especially in Brazil. One of the main factors that contributes to the lack of interest in the use of marijuana is the illicit recreational use of marijuana. But this is being released for cultivation and studies by competent companies, and may bring a series of benefits to both human health and pharmaceutical industries. It has numerous therapeutic actions related to secondary metabolites. It is worth highlighting the proven effects against diseases such as epilepsy, anxiety, depression, Parkinson's disease, multiple sclerosis and headache. For these reasons, in line with the world trend in which it is clear that consumers are prioritizing the use of naturalbased products, showing the wide field of study and technology for further study developments.
\end{abstract}

Keywords: Medicinal marijuana; Cannabis; Active principle; Herbal medicines; Integrative review.

\section{Resumen}

El objetivo del presente estudio fue exponer, a través de una revisión integradora de la literatura, la importancia del uso de principios activos derivados de la marihuana (Cannabis sativa) en la industria farmacéutica. Para este tipo de revisión integradora de la literatura se llevó a cabo. Se adoptó como criterio que incluya documentos de carácter científico, que presentaran una relación con la aplicación de la marihuana en la industria farmacéutica en un marco temporal entre 2006 y 2021, con el fin de discutir la evidencia científica de los últimos quince años. El uso de compuestos activos de Cannabis sativa en la industria aún es algo poco explorado, especialmente en Brasil. Uno de los principales factores que contribuye a la falta de interés por el uso de la marihuana es el uso recreativo ilícito de la marihuana. Tiene numerosas acciones terapéuticas relacionadas con los metabolitos secundarios. Cabe destacar los 
efectos probados frente a enfermedades como la epilepsia, la ansiedad, la depresión, la enfermedad de Parkinson, la esclerosis múltiple y el dolor de cabeza. Por estos motivos, en línea con la tendencia mundial en la que es evidente que los consumidores están priorizando el uso de productos de base natural, mostrando el amplio campo de estudio y tecnología para futuros desarrollos de estudios.

Palabras clave: Marihuana medicinal; Cannabis; Principio activo; Hierbas medicinales; Revisión integradora.

\section{Introdução}

As indústrias farmacêuticas dispõem de vastos investimentos em pesquisas para introduzir no mercado medicamentos inovadores usados para o tratamento, prevenção e diagnósticos de doenças, o qual apesar de seus efeitos adversos obtém resultados positivos, além de sua função social, a indústria farmacêutica possibilita a comercialização e distribuição dessas inovações visando a aquisição de lucros. Com esse intuito, a espécie vegetal Cannabis sativa L. se tornou objeto de estudo devido conter em sua composição química compostos eficientes para o tratamento de doenças (de Oliveira, et al, 2018).

No ano de 2017, no Brasil, a Agência Nacional de Vigilância Sanitária (ANVISA) incorporou a Cannabis sativa como planta medicinal na lista das Denominações Comuns Brasileiras (DCB) por meio da resolução de diretoria colegiada RDC No 156, de 5 de maio de 2017. Tal lista contém várias substâncias, plantas medicinais e princípios ativos de grande relevância para a indústria farmacêutica no país. Isso não quer dizer que a medida modificou as regras referente a maconha no Brasil, mas oficializa a Cannabis Sativa como planta medicinal e/ou como insumo de medicamentos com registro, por meio de algumas pesquisas científicas realizadas (Brasil, 2017). Nesse mesmo ano, no Brasil, foi registrado o primeiro medicamento derivado da Cannabis, o Mevatyl, indicado para o tratamento de espasmos moderados ou graves devido a esclerose múltipla (EM).

A Cannabis sativa L., é um vegetal que já vem sendo estudado a muitos anos, porém no Brasil ainda não se tem a utilização em proporções compatíveis com os diversos efeitos terapêuticos benéficos à saúde humana, obtidos a partir da maconha por meio dos compostos presentes na mesma. Os benefícios advindos por meio de seu uso são conhecidos a muito tempo pela China e Índia. Essa planta popularmente conhecida como maconha, é um arbusto, pertencente à família Moraceae, a qual se expande espontaneamente em diversas partes do mundo, dando ênfase às regiões tropicais e temperadas (Honório; Arroio \& Silva, 2006).

De acordo com Hall e Weier (2015), a utilização da Cannabis sativa vem aumentando mundialmente, o que leva os pesquisadores a uma discussão em relação a mesma. Além disso, ela ocupa o terceiro lugar em substâncias de maior uso em todo o território mundial.

Estudos demonstram que a maconha, foi de grande ajuda em pacientes portadores do vírus HIV, e gerou efeitos satisfatórios em tratamentos pós-quimioterápicos, isso devido ao efeito similar ao da morfina, ou seja, efeito sedativo. Além de efeitos benéficos para a saúde humana, a planta também apresenta efeitos tóxicos, que podem acometer o comportamento e a mente de seus usuários, tudo depende da dose utilizada, regra essa para todas as drogas existentes (Gonçalves \& Schlichting, 2014).

Efeitos adversos, de como a ocorrência de alucinações, sonolência pensamentos anormais, são propícios de um componente encontrado na planta, o $\Delta$-tetrahidrocanabidiol. Ademais há o Canabidiol (CBD) presente na maconha, que apresenta ações terapêuticas, muito utilizadas em ocasiões de ansiedade, epilepsia e anticonvulsivante, contudo, este composto auxilia no tratamento de distúrbios do sono, e é um anti-inflamatório (Pernoncini \& de Oliveira, 2014). Além de seu uso com fins terapêuticos, há o uso da maconha em rituais religiosos, alimentação, práticas medicinais e o seu uso recreativo.

É grande o interesse por composto canabinóides, uma gama de indústrias tem interesse em seu potencial terapêutico, alguns pesquisadores já produziram fármacos fundamentados em sua estrutura, levando a resultados um pouco controversos, devido ao impasse de isolar os efeitos psicotrópicos, o que inviabilizou o uso para fins medicinais dos compostos (Honório; 
Arroio \& Silva, 2006).

Tendo em vista a importância terapêutica e a recente ascensão do uso da maconha para fins medicinais o presente estudo tem por objetivo expor por meio de uma revisão integrativa da literatura a importância do uso dos princípios ativos advindos da maconha (Cannabis sativa) na indústria farmacêutica.

\section{Metodologia}

Uma revisão integrativa de literatura foi realizada, para tal fez-se busca com documentos científicos em distintas bases de dados tais como: Scielo, Medline (PubMed), Lilacs, Scopus, ScienceDirect, Periódicos Capes e em repositórios científicos. Adotou-se como critério que incluam documentos de cunho cientifico, nos idiomas português, inglês e espanhol, que apresentou relação com a aplicação da maconha na indústria farmacêutica em um recorte temporal entre 2006 a 2021, como intuito em discorrer sobre as evidências científicas dos últimos quinze anos. Também foram incluídos documentos legislatórios (governamentais) por sua vez tais não passaram por seleção prévia de datas para busca. Excluíram-se documentos os quais não apresentaram relação com os objetivos da pesquisa, por meio da leitura de título e resumo. Foram utilizadas as seguintes palavras-chave para busca dos arquivos científicos: Maconha; Maconha medicinal; Cannabis sativa; Cultivo; Produção; Legalização da maconha.

\section{Resultados e Discussão}

\section{Compostos ativos da maconha}

Há mais de 400 compostos químicos que se pode encontrar na maconha, estes são derivados de seu metabolismo secundário. Em meio a essas substâncias, existem 60 tipos de canabinóides, os quais estão entre eles canabidiol, canabinol, canabicromeno e THC (Tetra-hidrocanabinol) ilustrados na Tabela 1, porém os mais utilizados a fim medicamentoso, devido suas comprovações terapêuticas testadas cientificamente e que possuem ação nos receptores canabinóides, são o THC e o canabidiol (Nascimento; Dalcin, 2019).

Os canabinoides são compostos retirados da Cannabis sativa. Alguns estudos reúnem informações importantes sobre a utilização terapêutica dos derivados canabidiol (CBD) e delta-9-tetrahidrocanabinol (THC $\Delta$-tetrahidrocanabinol). O CBD esta incluída nessa variedade de substâncias encontradas na planta, representando mais de $40 \%$ do extrato (Ribeiro et al., 2021). Os autores ainda relatam que é diferente do THC - principal canabinoide psicoativo da maconha, visto que o CBD não causa euforia nem intoxicação. O THC é extraído do composto ácido tetrahidrocanabinólico (THCA) forma inativada, sem apresentar efeitos psicoativos, embora possuam propriedades neuroprotetoras. Este composto é a forma mais ativa de canabinoide resultando em alterações características de humor e percepção.

A obtenção de compostos canabinóides na forma sintetizada desperta uma grande atração para os laboratórios e indústrias. Determinadas indústrias e até mesmo laboratórios acadêmicos utilizam esses compostos canabinóides para desenvolver medicamentos fundamentados nas estruturas destes (Honório; Arroio \& Silva, 2006).

Os canabinóides dividem-se em três tipos, os quais são fitocanabinóides, canabinóides sintéticos e os canabinóides endógenos ou endocanabinóides, essas são substâncias químicas naturais, são caracterizadas especialmente pela anandamida ( $\mathrm{N}$-araquidonoil etanolamina) e pelo 2-araquidonoil glicerol. Eles estão fisiologicamente relacionados à função de comer, relaxar, dormir, esquecer e proteger (Lessa; Cavalcanti \& Figueiredo, 2016). 
Tabela 1 - Principais canabinóides encontrados na maconha.

\begin{tabular}{|c|c|c|}
\hline Canabinóides & Indicações terapêuticas & Estrutura química \\
\hline Canabidiol (CBD) & $\begin{array}{l}\text { Não apresenta propriedades psicoativas, } \\
\text { indicado para doenças } \\
\text { neurodegenerativas como Doença de } \\
\text { Parkinson, dores neuropáticas, } \\
\text { Esclerose Múltipla, antioxidante. }\end{array}$ & \\
\hline$\Delta 9$-tetrahidrocanabinol & $\begin{array}{l}\text { Analgésico, ansiolítico, } \\
\text { anticonvulsionante. }\end{array}$ & \\
\hline Canabicromeno & $\begin{array}{l}\text { Não apresenta propriedades psicoativas, } \\
\text { mas possui funções anti-inflamatórias e } \\
\text { antimicrobianas. }\end{array}$ & \\
\hline Canabinol & $\begin{array}{l}\text { São subprodutos de isolação } \\
\text { provenientes da aromatização oxidativa } \\
\text { do } \Delta 9 \text {-THC }\end{array}$ & \\
\hline
\end{tabular}

Fonte: Adaptado de Borille (2016); Graça (2020).

Os fitocanabinoides comportam-se de forma irregular na região cerebral, especialmente em receptores CB1, o que ocasiona efeitos terapêuticos estigmáticos conhecidos como "tétrade" de efeitos canabinóides, o que envolve efeitos de analgesia, hipotermia, sedação e catalepsia, basicamente em doses elevadas. Os diversos efeitos dos fitocanabinoides são entrepostos por ações agonistas ou antagonistas de receptores específicos (Franco \& Viegas, 2017).

Os canabinóides sintéticos ainda apresentam complexidade, pois suas estruturas demonstram formas diversas e sua farmacologia ainda possui incógnita, além disso, eles são representados como um problema de saúde pública. Esses canabinóides sintéticos manifestaram-se como drogas de abuso. O mecanismo de ação dessas substancias baseiam-se no efeito agonista de CB1 especificamente compreensível, o que se mostra bastante eficaz, e ocasiona efeitos narcótico, alucinógeno, depressor e causa dependência (Franco \& Viegas, 2017).

As substâncias endocanabinoides são sintetizadas por demanda no sistema nervoso central (SNC) com o intuito de diminuir a sensibilidade à dor. Eles parecem ser mediadores comprometidos na modulação de acontecimentos dolorosos como o wind up e a alodinia. Através de mecanismos mediadores por receptores $\mathrm{CB}^{1}$ e $\mathrm{CB}^{2}$, o sistema endocanabinóide tem papel importante também na dor periférica, principalmente em processos inflamatórios e no caso de hiperalgesia. O THC inibe a 
síntese de prostaglandinas E-2 e impulsiona a lipoxigenase, todavia não interfere na síntese de cicloxigenase-1 e cicloxigenase2. Os canabinóides também apontam uma atividade antioxidante via mecanismo não canabinóides (Lessa; Cavalcanti \& Figueiredo, 2016).

\section{Compostos canabidioicos}

O canabidiol (CBD) é um composto identificado nas flores da planta. Ele pertence ao grupo dos terpenofenólicos, dentre as diversas funcionalidades do canabidiol, podemos citar o seu alívio em sintomas de dores, náusea, ansiedade, inflamação e distúrbios neurológicos. É valido ressaltar que este dispõe de uma série de outras vantagens para a saúde humana (Neri et al., 2018).

A aplicabilidade do CBD tem sido mais aceita, do que os demais canabinoides, devido a sua capacidade de não causar intoxicações iguais às do THC (que levam o paciente a desenvolver formas agudas e temporárias de ansiedade, acometidos por doses que não compreendem a capacidade de aceitação do organismo), as quais muitas das vezes são comparadas a taques de pânico. Como se sabe que a intoxicação é um efeito colateral grave, o CBD se torna mais aceito, devido a não apresentar essas reações. A confiabilidade em relação a esse composto tem aumentado, isso porque os médicos substituem outros opiáceos por CBD, de modo que este está sendo mais confiável (Neri et al., 2018).

Outro composto canabidióico encontrado na maconha e de importância terapêutica é o THC, este é responsável pelos efeitos alucinógenos, além de ser o componente principal da Planta. O THC, é excretado na resina do vegetal feminino para que haja proteção da flor contra o sol. Então o THC é obtido da resina, que é um aliado do poder de proteção da planta, além de ser um excelente herbicida (Gonçalves \& Schlichting, 2014).

Não é de hoje que o THC vem sendo estudado, pesquisas relatam que esses apresentam efeitos sedativos e hipnóticos em diversos quadros clínicos, como esclerose múltipla. Além disso, ele se torna promitente à síndrome de abstinência à cannabis. Mas, além de efeitos benéficos, há os efeitos negativos, permitindo ao usuário um grau de dependência a essas substâncias (Crippa; Zuardi \& Hallak, 2010).

\section{Problemas de inserção da maconha na sociedade}

O choque de dúvidas em relação aos males a saúde que a maconha pode causar ainda é muito grande, não se há ocorrências de overdoses decorrente do uso da mesma, mas os estudos refletem que as mortes lentas são causadas simultaneamente pelo seu uso e a todo o volume de violências e crimes ligados ao uso de drogas inclusive da maconha (Ribeiro, 2014).

A aprovação plena da Cannabis é um fator de complexidade, a mesma ainda precisa passar por inúmeros estudos e comprovações rigorosas, para enfim ser aceita tanto na sociedade, quanto por lei. Para essa aceitação há a necessidade do envolvimento político, económico, cultural e social (Ribeiro, 2014).

O desempenho social ao uso da maconha adquire significados conforme os grupos respectivos ao qual se inserem. Esses significados são resultantes da observação popular e conhecimento cultural sobre suas propriedades farmacológicas, diante disso, há uma série de julgamentos populares, adquiridos por comunicações formais e informais, sendo estes julgados e reelaborados socialmente (Silva et al., 2018). Os mesmos autores ainda relatam que a população que não possui acesso aos significados cabíveis em relação a aplicação da maconha, e que detém apenas o conhecimento popular, podem expor pensamentos inadequados sobre a mesma, levando a preconceitos desvirtuados, em vista que o senso comum não traz conceitos abrangentes ao verdadeiro potencial encontrado na $C$. sativa.

A sociedade tem um foco maior no lado prejudicial da maconha, na forma coercitivo de ingerir, mas, se for levado em consideração essa mesma conclusão em drogas licitas, não haveria possibilidade de ingerir nenhuma substancia química no uso 
farmacológico, levando-se em conta que as mesmas, podem ser devidamente prejudiciais à saúde, usadas de forma incorreta e exacerbadas, causando até intoxicações severas (Silva et al., 2018).

\section{Uso da maconha na indústria}

A inovação de produtos, que trazem melhorias é o elemento principal no padrão de competição da indústria, isso é possível devido os avanços tecnológicos, os quais exigem investimentos elevados em Pesquisa e Desenvolvimento (P\&D). Tal processo conta com um enorme respaldo do sistema internacional de propriedades intelectuais. Diante disso, a indústria farmacêutica vem buscando novos princípios ativos para inovar o mercado de medicamentos, além disso, ela busca o aprimoramento de produtos já existentes, com intuito de trazer benefícios para a saúde como prevenção, diagnóstico, tratamento e cura de diversas doenças. Na tentativa de trazer a cura, a indústria farmacêutica consegue obter diversos lucros através desses aprimoramentos (de Oliveira et al., 2018).

As descobertas obtidas por meio de inúmeras pesquisas estimularam a realização de estudos mais avançados usando o CBD em atividades farmacológicas, nos quais, verificou-se que o composto possuía ações farmacológicas parecidas ao do composto encontrado na Cannabis, o Delta-9-THC; e que o canabidiol possuía efeito sedativo e antipsicótica, aspectos que gerou expectativas em relação a sua utilização no tratamento dos sintomas de esquizofrenia (de Oliveira et al., 2018).

O canabidiol, que é um dos componentes mais promissores da planta (Maconha), atualmente encontra-se fora da lista de substâncias proibidas no Brasil pela ANVISA, a mesma foi inserida na lista de substâncias controladas C1 da Portaria/SVS no 344, de 12 de maio de 1998 (Brasil, 1998). A ANVISA contemporaneamente deu-se a aprovação de importação de medicamentos que haja em seus componentes substâncias originárias da Cannabis sativa. Isso ocorreu devido a retirada do canabidiol da relação de substâncias proibidas e sua inserção junto às substâncias de uso controlado. Essa conclusão foi tomada devido à alta quantidade de pesquisas e ações judiciais que favoreceram a aplicação de remédios com esse composto nos casos de epilepsia. Devido a isso, possibilitará a ingressão de extratos padronizados produzidos por indústrias farmacêuticas internacionais para abordar casos graves da doença.

Recentemente, a ANVISA aprovou a Resolução de Diretoria Colegiada nº 325, de 3 de dezembro de 2019, a mesma renovou a lista de substâncias proibidas da Lista F da Portaria n $344 / 1998$, o que permitiu venda de medicamentos à base de Cannabis sativa em território nacional (Brasil, 2019).

Dentro desta discussão é considerável salientar que as empresas brasileiras também produzem medicamentos à base de maconha e realizam pesquisas em torno das propriedades terapêuticas da planta. A Justiça Federal da Paraíba concedeu por meio de uma liminar de tutela provisória que a Associação Brasileira de Apoio à Cannabis Esperança - Abrace - cultive e manipule a erva para finalidades medicinais. Com isso, permitem que os associados à organização comprem os produtos dentro de algumas regras estabelecidas (Brasil, 2017). De acordo com Zaganelli e Correia (2018), outro aspecto são as atividades realizadas nos centros de ensinos no Brasil, a Universidade de São Paulo Campus Ribeiro Preto, a Fundação Oswaldo Cruz e a Universidade Federal do Rio de Janeiro, tem como objetivo o desenvolvimento de medicamentos e testes certificados em pacientes com problemas neurológicos e formam uma parceria para o desenvolvimento também a base de maconha. Além disso, iniciou o projeto Farmacannabis onde o mesmo investiga a composição dos remédios industrializados importados e produzidos artesanalmente.

\section{Aplicabilidade da maconha na indústria farmacêutica}

As plantas medicinais são aplicáveis como recursos terapêuticos a muitos anos, motivo dessa aplicação se da pela quantidade de princípios ativos encontrados nessas plantas, e outro fator recorrente disso é por serem economicamente mais acessíveis, além de trazer uma série de possibilidades as indústrias farmacêuticas (Lima et al, 2016). Com isso, uma 
espécie tem chamado muita atenção das indústrias farmacêuticas, que é a Cannabis sativa, já que a mesma possui propriedades terapêuticas para a saúde humana (de Oliveira et al., 2018).

As substâncias que são extraídas da Cannabis sativa são estudadas pela indústria farmacêutica mundialmente. Mas, o cultivo da mesma especialmente para as indústrias farmacêuticas acarreta uma alta complexidade na monitorização das variações dos metabolitos secundários em questão, e busca a aceitação de elevados padrões de desenvolvimento, que ainda lida com a confusão de informação sobre o assunto (Potter, 2014).

$\mathrm{Na}$ área da farmacologia encontra-se inúmeros desafios quanto ao desenvolvimento de estruturas químicas inovadoras que servirão como base para a produção de novos e eficazes agentes terapêuticos. Atualmente, as pesquisas realizadas a respeito dessa planta passaram a compreender de forma mais clara os mecanismos de ação que intermediam os efeitos amplamente conhecidos da mesma (Vieira; Marques \& Sousa, 2020).

Os medicamentos que tem como base os compostos canabinoides vem sendo considerado como uma escolha promissora para o tratamento de várias morbidades em diversos países. No Brasil, a inserção desses medicamentos ainda é recente e seus impactos sobre a saúde humana encontram-se em procedimentos de formulação no âmbito das políticas públicas. Com isso, mesmo havendo várias evidencias cientificas sobre o potencial terapêutico da planta, as famílias ou pacientes encontram diversos desafios para terem acesso as terapias medicamentosas a base de Cannabis (Vieira; Marques \& Sousa, 2020).

O primeiro medicamento obtido diretamente da planta C. Sativa foi sintetizado no laboratório britânico GW Pharmaceuticals. Depois de passar por testes clínicos e seguidamente ser aprovada para prescrição médica, sua utilização foi admitida pelo Canadá, onde é vendida na forma de spray oral e é conhecida comercialmente como Sativex®. É utilizado por pacientes com dor oncológica, neuropática e esclerose múltipla. Um outro medicamento liberado para uso no Canadá é a Nabilona (Cesamet $\left.{ }^{\circledR}\right)$, indicado para alívio da dor neuropática crônica e é antiemético em pacientes oncológicos (Carneiro, 2018).

Também há no mercado o THC sintético dranabinol, conhecido no mercado como Maritol, o qual diminuiu a pressão intra-ocular no glaucoma e é indicado para aumentar o apetite e conservar o peso de pacientes com síndrome de imunodeficiência adquirida (SIDA). O mesmo está disponível para utilização médica nos EUA desde 1985 (Carneiro, 2018).

\section{Medicamentos à base de Cannabis sativa}

Os compostos canabinóides possuem grande interesse por profissionais de saúde devido ao seu papel terapêutico que constantemente mostra-se considerável a adesão ao tratamento de diversas patologias (Ribeiro, 2014).

Os canabinóides ativos foram utilizados na medicina devido aos avanços tecnológicos na área da química e farmacologia, já que os mesmos foram obtidos na forma pura (Ribeiro, 2014). Nos anos anteriores foram sintetizados muitos compostos canabinóides, esses podem ser vistos na Tabela 2.

$\mathrm{Na}$ área experimental, há numerosos compostos canabinóides os quais seus potenciais terapêuticos estão em investigação. As classes englobam compostos que ativam e bloqueiam os canais dos receptores canabinóides, inibidores da recaptação e/ou degradação que avolumam os níveis endógenos dos canabinóides, os moduladores alostéricos que fazem regulação fina dos receptores, e extratos padronizados contendo fitocanabinoides (Pamplona, 2014).

Com a crescente quantidade de estudos acerca da Cannabis Sativa, várias publicações a respeito da mesma, apresentam concordância entre si e relatam que a planta apresenta efeitos terapêuticos em várias patologias. Os resultados são positivos para propriedade ansiolítica, antipsicótica, diabetes, HIV/AIDS, glaucoma, retinopatia diabética, câncer, dentre outras. Com isso, têm-se uma contribuição significativa para a mudança de cultura para aceitação da utilização da maconha como medicamento (Oliveira \& Lima, 2016). 
Research, Society and Development, v. 10, n. 12, e46101219829, 2021

(CC BY 4.0) | ISSN 2525-3409 | DOI: http://dx.doi.org/10.33448/rsd-v10i12.19829

Tabela 2 - Fármacos derivados dos canabinóides e seus referidos usos terapêuticos.

\begin{tabular}{|c|c|c|}
\hline Nome comercial & $\begin{array}{ll}\text { Denominação } & \text { comum } \\
\text { internacional (DCI) } & \end{array}$ & Utilização terapêutica \\
\hline Marinol® & Dronabinol & $\begin{array}{l}\text { Estimulação do apetite e antiemético em doentes } \\
\text { oncológicos e com síndrome de imunodeficiência } \\
\text { adquirida (SIDA). }\end{array}$ \\
\hline Cesamet ${ }^{\circledR}$ & Nabilona & $\begin{array}{l}\text { Estimulação do apetite e antiemético em doentes } \\
\text { oncológicos e com SIDA. }\end{array}$ \\
\hline Sativex ${ }^{\circledR}$ & THC e Canabidiol & $\begin{array}{l}\text { Tratamento da rigidez muscular e dor neuropática em } \\
\text { doentes com Esclerose Múltipla; Analgésico em } \\
\text { doentes oncológicos terminais. }\end{array}$ \\
\hline Acomplia ${ }^{*}$ & Rimonabant & $\begin{array}{l}\text { Redução do apetite; } \\
\text { Tratamento da obesidade }\end{array}$ \\
\hline
\end{tabular}

Nota: *Autorização e comercialização suspensa em outubro de 2008.

Fonte: Ribeiro, (2014).

\section{Patologias tratadas com medicamento obtidos da maconha}

Ao longo da história a $C$. sativa vem sendo utilizada como base para medicamentos. Acha-se registros de uso da planta como insumo medicinal na China antiga, usada para diversos problemas, tanto no seu uso in natura, como a manipulação para uso dos derivados, a maconha esteve presentemente como subsídio em diversos tratamentos. O uso tradicional começou a ser conduzido para o campo da ciência, com o objetivo de se comprovar cientificamente suas propriedades terapêuticas positivas no combate das patologias (Zaganelli \& Correia, 2018). Destaca-se o tratamento com uso da maconha para epilepsia, ansiedade, depressão, doença de Parkinson, esclerose múltipla e cefaleia.

\section{Epilepsia}

Pacientes epiléptico são resistentes ao tratamento com medicamentos anticonvulsivantes, apesar do médico escolher a terapêutica certa usando doses convenientes e toleradas pelo paciente. A qualidade de vida do paciente é comprometida quando as crises epilépticas não são controladas, e são relacionadas à comorbidade médicas, psicossocial e cognitivas (Oliveira \& Lima, 2016).

Em pacientes com síndromes epiléticas heterogêneas o CDB tem mostrado papel terapêutico muito importante para aquisição desses, pacientes cuja já passaram por outros meios de tratamento e puderam ter o diagnostico de intratáveis ou de difícil controle, o que os trouxeram inúmeros efeitos colaterais ao ingerir outros fármacos (Ribeiro et al, 2021).

\section{Ansiedade e depressão}

Estudos confirmados em animais revelam que o canabidiol é um composto seguro para a utilização em seres humanos. Os estudos realizados em ratos e camundongos mostram a eficácia do efeito ansiolítico do canabidiol utilizando uma dose de $20 \mathrm{mg} / \mathrm{kg}$. Esses efeitos não parecem ser mediados pelos receptores benzodiazepínicos, por outro lado, este canabinóide interage com os receptores 5-HT1A e esta interação parece estar envolvida em sua ação ansiolítica (Crippa; Zuardi \& Hallak, 2010). Além disso, os autores também abordam sobre um estudo realizado em humanos saudáveis com o CBD (via oral, inalatória ou endovenosa), em um método de simulação do falar em público (SFP), onde foram comparados o canabidiol (300 mg), o placebo e dois ansiolíticos, o diazepem $(10 \mathrm{mg}$ ) e a ipsapirona (5 mg) num método duplo cego. A pesquisa ainda 
expos que tanto o canabidiol (CBD) como os dois ansiolíticos diminuíram a ansiedade induzida pela simulação do falar em público (SFP), e não houve efeito colateral significante.

\section{Doença de parkinson}

Apesar de ainda haver pouco estudo na literatura sobre o tratamento da doença de Parkinson, estudos atuais mostram um efeito terapêutico do canabidiol nesta patologia. Estudos constatam que a relação da doença de Parkinson com os canabinóides está na neuroproteção, as propriedades antioxidantes do canabidiol podem fornecer proteção contra a degeneração progressiva dos neurônios dopaminérgicos da região nigro-estriatal, característica da doença de Parkinson (Oliveira \& Lima, 2016).

\section{Esclerose múltipla}

A utilização de alguns componentes da Cannabis sativa é bastante discutida no tratamento sintomático e preventivo da esclerose múltipla (EM). O medicamento naxibimol é utilizado em alguns países, indicado especificamente para espasticidade na EM, onde possuem THC e CRB, na proporção de 1:1, de uso oro-bucal. Para o tratamento da espasticidade, estudos mostram melhora nas escalas de auto avaliação em algumas semanas. Na dor neuropática ou central estudos realizados identificaram eficácia variável, já no tratamento de tremores e disfunção vertical o medicamento demonstrou-se ineficaz (Brucki et al., 2015).

\section{Cefaleia}

Apesar de não existirem estudos recentes na cefaleia, patologias relacionadas à dor do segmento cefálico responderam a utilização do canabinóides, como na dos neuropáticos orofacial e sua ação no sistema de dor central onde apresentou muita intersecção com vias dolorosas relacionadas nas dores de cabeça (Brucki et al., 2015). Porém, recentemente Carneiro e Morgadinho (2019) afirmaram que, mesmo diante de alguma evidência existente o benefício da utilização terapêutica da maconha medicinal como profilático de enxaqueca e outras cefaleias crónicas está distante de estar claramente estabelecido. Deste modo, ainda há uma divergência em torno da aplicação da Maconha medicinal como tratamento para cefaleia.

\section{Benefícios e malefícios da utilizaçao da maconha}

Há centena de anos a maconha vem sendo utilizada para fins medicinais. No entanto, antigamente não havia técnicas ou instrumentos científicos que pudessem confirmar tais propriedades de seus princípios ativos. Atualmente, várias propriedades medicinais da planta já foram confirmadas cientificamente. Esses princípios ativos, ajuda na cura de pacientes com câncer, AIDS, glaucoma entre outras doenças. Em alguns países, como o Canadá, já existe um spray bucal com extratos da Maconha para auxiliar no tratamento de pacientes com esclerose múltipla (Luz Filho; Albuquerque, 2018).

O composto canabidioico, compreende habilidade antipsicótica mediante sua disponibilidade de anandamida, além de ser auxiliador na sinalização dos endocanabinóides, diante do impedimento da recaptação da anandamida. Além disso, o CBD pode ser utilizado no tratamento de doenças como a epilepsia, artrite reumatoide, doença de Parkinson, doença de Alzheimer, ansiedade e depressão (Alves, 2021).

Destaca-se que todas estas doenças devem ser diagnosticadas, medicadas e tratadas, de maneira correta, pois o uso indiscriminado da Cannabis Sativa não é benéfico e pode ocasionar algumas complicações, especialmente se administrado de forma errada. Com isso, alguns efeitos colaterais aparecem juntamente as possibilidades terapêuticas como alterações na memória e cognição, depressão, efeito sedativo, euforia, dentre outros (Zaganelli \& Correira, 2018). Os autores relatam ainda que outro ponto importante é sobre os efeitos negativos do uso da Cannabis. Essa restrição existente se explica pelo aspecto 
entorpecente encontrado na planta. A sua utilização in natura e sob a utilização na forma de fumo geram malefícios à saúde dos usuários, como quadros clínicos de psicose.

Segundo a Associação Brasileira Multidisciplinar de Estudos sobre Drogas (ABRAMD, 2006), a maconha é a droga ilícita mais consumida no mundo, sendo a primeira da lista em vários países. Apesar disso, não há relatos de casos de morte por "overdose" da droga - ou seja, mesmo apresentando diversos efeitos no nosso organismo, a maconha não apresenta riscos de mortes a seus usuários, apesar de seu uso excessivo acarretar complicações psíquicas por déficit de função, atingindo algumas áreas do cérebro.

De acordo com Honório, Arroio e Silva (2006), os efeitos adversos da Cannabis podem ser divididos em 2 categorias: Os efeitos do hábito de fumar a planta que provoca alterações das células do trato respiratório e aumenta a incidência de câncer de pulmão entre os usuários, e os causados pelas principais substâncias isoladas (canabinóides). Um dos efeitos associados ao longo do tempo de exposição aos canabinóides é a dependência causada principalmente pelos efeitos psicoativos com a cessação do uso. Irritabilidade, insônia, agitação, náusea e câimbras são sintomas da dependência dos efeitos psicotrópicos da maconha Pesquisas também mostram que a Cannabis não causa dependência física e que a suspensão do uso não causa síndrome de abstinência. Seu uso prolongado em certas circunstâncias causa dependência psicológica, e pode levar ao consumo de outras drogas. Por ser uma poderosa droga psicotrópica e alucinogênica, seu uso indiscriminado é perigoso.

Muitos dos efeitos positivos da utilização da maconha são amplamente divulgados e ricamente provados, mas os seus efeitos negativos ainda permeiam os diálogos sobre sua legalização para fins terapêuticos e até mesmo em países onde já se utilizam medicamentos derivados de elementos isolados da planta (Penha et al., 2019).

\section{Considerações Finais}

A utilização dos compostos ativos da Cannabis sativa na indústria ainda é algo pouco explorado, principalmente no Brasil. Um dos principais fatores de que contribui para que não haja maior interesse em torno da utilização da maconha é o uso recreativo ilícito da mesma. Mas esta está sendo liberada para cultivo e estudos de empresas competentes, podendo trazer uma série de benefícios tanto a saúde humana, quando para as indústrias farmacêuticas. A mesma apresenta numerosas ações terapêuticas relacionadas aos metabolitos secundários. É valido ressaltar os efeitos comprovados frente a doenças como epilepsia, ansiedade, depressão, doença de Parkinson, esclerose múltipla e cefaleia. Por esses motivos em concordância com a tendência mundial a qual é notório que os consumidores estão priorizando o uso de produtos à base natural (fitoterápicos), mostrando o amplo campo de estudo e tecnológicos para desenvolvimentos de estudos posteriores

\section{Referências}

Alves, F. E. F. (2020). A utilização medicinal do canabidiol como recurso terapêutico: revisão bibliográfica. Revista Interfaces: Saúde, Humanas e Tecnologia, 8(2), 581-590. https://doi.org/10.16891/2317-434X.v8.e2.a2020.pp581-590

Associação Brasileira Multidisciplinar de Estudos sobre Drogas (ABRAMD). Maconha: Uma visão multidisciplinar 2006. http://www.neip.info/downloads/textos\%20novos/maconha.pdf

Borille, B. T. (2016). Caracterização química da planta Cannabis sativa L. a partir de sementes apreendidas pela policia federal no estado do Rio Grande do Sul. Tese (Doutorado em ciências farmacêuticas) - Universidade federal do Rio Grande do Sul da faculdade de farmácia, Porto Alegre. http://hdl.handle.net/10183/159507

Brasil. (1998). Portaria n 344, de 12 de maio de 1998. Aprova o Regulamento Técnico sobre substâncias e medicamentos sujeitos a controle especial. Diário Oficial da União.

Brasil (2017). 2 $2^{\mathrm{a}}$ Vara Federal - PB. Processo $\mathrm{n}^{\mathrm{o}}$ : 0800333-82.2017.4.05.8200, AUTOR: ASSOCIACAO BRASILEIRA DE APOIO CANNABIS ESPERANCA - ABRACE. Advogado: Yvson Cavalcanti De Vasconcelos e outro. Réu: Agência Nacional de Vigilância Sanitária e outro. Relator: Juíza Wanessa Figueiredo dos Santos Lima. Diário Oficial da União. 
Research, Society and Development, v. 10, n. 12, e46101219829, 2021

(CC BY 4.0) | ISSN 2525-3409 | DOI: http://dx.doi.org/10.33448/rsd-v10i12.19829

BRASIL. Ministério da saúde. Agencia Nacional de Vigilância Sanitária (2019). Resolução da Diretoria Colegiada $n^{\circ} 325$, de 3 de dezembro de 2019. Dispõe sobre a atualização do Anexo I (Listas de Substâncias Entorpecentes, Psicotrópicas, Precursoras e Outras sob Controle Especial) da Portaria SVS/MS n 344, de 12 de maio de 1998. Diário Oficial da União.

BRASIL. Ministério da Saúde. Agência Nacional de Vigilância Sanitária (2017). Resolução da Diretoria Colegiada ${ }^{\circ}$ 156, de 5 de maio de 2017. Dispõe sobre a alteração das Resoluções da Diretoria Colegiada - RDC n ${ }^{\circ}$ 64/2012, $n^{\circ}$ 29/2013, $n^{\circ} 42 / 2014, n^{\circ} 1 / 2015, n^{\circ} 11 / 2015, n^{\circ} 71 / 2016$ e $n^{\circ} 104 / 2016, p^{2}$ ara a inclusão, alteração e exclusão de Denominações Comuns Brasileiras - DCB, na lista completa das DCB da Anvisa. Diário Oficial da União.

Brucki, S., Frota, N. A., Schestatsky, P., Souza, A. H., Carvalho, V. N., Manreza, M. L. G., Mendes, M. F., Comini-Frota, E., Vasconcelos, C., Vitor Tumas, V., Ferraz, H. B., Barbosa, E., \& Jurno, M. E. (2015). Cannabinoids in neurology-Brazilian Academy of Neurology. Arquivos de Neuro-psiquiatria, 73, 371374. https://doi.org/10.1590/0004-282X20150041

Carneiro, D. A. (2018). USO MEDICINAL DE Cannabis sativa. Monografia (Bacharel em Direito), UniEVANGÉLICA. http://45.4.96.19/bitstream/aee/562/1/Monografia\%20-\%20Daniel\%20Alves.pdf

Carneiro, D. R., \& Morgadinho, A. S. (2019). Canábis Medicinal na Neurologia Clínica: Uma Nuvem de Incertezas. Sinapse, 19(3-4), 104-113.

Crippa, J. A. S., Zuardi, A. W., Hallak, J. E. (2010). Uso terapêutico dos canabinoides em psiquiatria. Braz. J. Psychiatry. 32: 556-566. https://doi.org/10.1590/S1516-44462010000500009

de Oliveira, E. H. A., Sorgato, J. P. A., Silva, R. C., da Silva Martins, P. K., Uchôa, S. B. B., \& Tonholo, J. (2018). Mapeamento Tecnológico do Canabidiol (CBD) para Finalidades Farmacêuticas no Brasil. Cadernos de Prospecção, 11(3), 900.

Gonçalves, G. A. M., \& Schlichting, C. L. R. (2014). Efeitos benéficos e maléficos da Cannabis sativa. Revista UNINGÁ Review, 20(1). http://revista.uninga.br/index.php/uningareviews/article/view/1560

Gontijo, É. C., Castro, G. L., de Castro Petito, A. D., \& Petito, G. (2016). Canabidiol e suas aplicações terapêuticas. Revista Eletrônica da Faculdade de Ceres, 5(1). https://doi.org/10.36607/refacer.v5i1.3360. Acesso em: 3 fev. 2021.

Graça, M. C. S. (2020). Canabinóides: estrutura química, efeitos farmacológicos e utilização terapêutica. Dissertação (Mestrado em ciências farmacêuticas), instituto universitário Egas Moniz.

Hall, W., \& Weier, M. (2015). Assessing the public health impacts of legalizing recreational cannabis use in the USA. Clinical pharmacology \& therapeutics, 97(6), 607-615. https://doi.org/10.1002/cpt.110

Honório, K. M., Arroio, A., \& Silva, A. B. F. D. (2006). Aspectos terapêuticos de compostos da planta Cannabis sativa. Química nova, 29 , 318-325. https://doi.org/10.1590/S0100-40422006000200024

Lessa, M. A., Cavalcanti, I. L., \& Figueiredo, N. V. (2016). Derivados canabinóides e o tratamento farmacológico da dor. Revista Dor, 17, 47-51. https://doi.org/10.5935/1806-0013.20160012

Lima, A. M. C. Santos, P. M. A. Carvalho, A. A. Santos, W. C. (2016). Uso do $\Delta 9$-tetraidrocanabinol: um estudo de prospecção tecnológica. Revista geintecgestao inovacao e tecnologias, 6(3), 3367-3376. D.O.I.: 10.7198/S2237-072220160003010.

Luz Filho, A. F., \& Albuquerque, S. A. F. (2018). USO DA MACONHA COMO MEDICAMENTO. Revista de Ensino e Cultura. 01(02), 116

Nascimento, A. G. T. P., \& Dalcin, M. F. (2019). Uso terapêutico da Cannabis sativa: uma breve revisão. Brazilian Journal of Surgery and Clinical Research $-B J S C R, 27(2), 164-169$.

Neri, M., Nascimento, C., Gonçalvez, R., \& Noronha, T. (2018). Entre a ciência e a insegurança. Revista do Farmacêutico, 135(1), $32-39$.

Oliveira, K. L. $\quad$ B., \& Lima, T. P. S. (2016). Cannabis sativa: potencial terapêutico http://repositorio.saolucas.edu.br:8080/xmlui/bitstream/handle/123456789/1710/Kauanna\%20Lamartine\%20Brasil\%20Oliveira\%20-

$\% 20$ Cannabis\%20sativa\%20-\%20potencial\%20terap\%C3\%AAutico.pdf?sequence=1

Pamplona, F. A. (2014). Quais são e pra que servem os medicamentos à base de Cannabis. Revista da biologia, 13(1), 28-35. https://doi.org/10.7594/revbio.13.01.05

Penha, E. M., Cardoso, D. D., Coelho, L. P., \& Bueno, A. M. (2019). A regulamentação de medicamentos derivados da Cannabis sativa no Brasil. Brazilian Journal of Forensic Sciences, Medical Law and Bioethics, 9(1), 125-145. http://dx.doi.org/10.17063/bjfs9(1)y2019125

Pernoncini, K. V., \& Oliveira, R. M. M. W. (2014). Usos terapêuticos potenciais do canabidiol obtido da Cannabis sativa. Revista Uningá Review, 20(3).

Potter, D. J. (2014). A review of the cultivation and processing of cannabis (Cannabis sativa L.) for production of prescription medicines in the UK. Drug testing and Analysis, 6(1-2), 31-38. https://doi.org/10.1002/dta.1531

Ribeiro, R. G., Nery, L. G., Costa, A. C. M. M., Oliveira, G. S. Vaz, R. L., Fontoura, H. S., Arruda, J. T. (2021). Potencial uso terapêutico dos compostos canabinoides - canabidiol e delta-9-tetrahidrocanabidiol. Research, Society and Development. 10 (4). DOI: http://dx.doi.org/10.33448/rsd-v10i4.13844.

Ribeiro, J. A. C. (2014). A Cannabis e suas aplicações terapêuticas. Dissertação (Mestrado em Ciências Farmacêuticas) Universidade Fernando Pessoa. http://hdl.handle.net/10284/4828

Silva, A. S., Gomes, J., Palhano, M. B., \& Arantes, A. C. Y. (2018). A maconha nas perspectivas contemporâneas: benefícios e malefícios: Imagem: StockPhotos. Revista Científica da Faculdade de Educação e Meio Ambiente, 9(2), 786-795. http://dx.doi.org/10.31072/rcf.v9i2.670 
Research, Society and Development, v. 10, n. 12, e46101219829, 2021

(CC BY 4.0) | ISSN 2525-3409 | DOI: http://dx.doi.org/10.33448/rsd-v10i12.19829

Viegas Jr, C., \& Franco, G. D. R. R. (2017). A contribuição de estudos do canabidiol e análogos sintéticos no desenho de novos candidatos a fármacos contra transtornos neuropsiquiátricos e doenças neurodegenerativas. Revista Virtual de Química, 9(4).

Vieira, L. S., Marques, A. E. F., \& de Sousa, V. A. (2020). O uso de Cannabis sativa para fins terapêuticos no Brasil: uma revisão de literatura. Scientia Naturalis, 2(2). https://periodicos.ufac.br/index.php/SciNat/article/view/3737

Zaganelli, M. V., \& Correia, J. V. G. (2018). A restrição do uso medicinal da cannabis sativa face ao princípio da autonomia da vontade. Revista Eletrônica do Curso de Direito da UFSM, 13(2), 611-639. http://dx.doi.org/10.5902/1981369429501 